\title{
A Retrospective Large Original Study of Cutaneous Melanoma
}

\author{
HOJAT EFTEKHARI ${ }^{1}$, SHABNAM FAHIM ${ }^{2,3}$, ZEINAB ARYANIAN ${ }^{3}$, AZADEH GOODARZI $^{4}$, ALI SADEGHINIA*3, PEDRAM \\ NOURMOHAMMADPOUR ${ }^{3}$, MONIREH MAZANDARANI ${ }^{5,6}$, ALI POURALI ${ }^{7}$, SEPEHR KHOSRAVI8,9, ZOHRE \\ KHODASHENAS 3 , MAISA NOURALINEJAD ${ }^{3}$ \\ ${ }^{1}$ Department of Dermatology, Guilan University of Medical Sciences, Rasht, Iran \\ ${ }^{2}$ Department of Dermatology, Pediatric Dermatology Fellowship, Razi Hospital, Tehran University of Medical Sciences, Tehran, Iran \\ ${ }^{3}$ Autoimmune Bullous Disease Center, Razi Hospital, Tehran University of Medical Sciences, Tehran, Iran \\ ${ }^{4}$ Department of Dermatology, Rasool Akram Medical Complex, Iran University of Medical Sciences, Tehran, Iran \\ ${ }^{5}$ Department of General Medicine, Shahroud University of Medical Sciences, Shahroud, Semnan, Iran \\ ${ }^{6}$ Golestan University of Medical Sciences, Sayad Shirazi Hospital, Gorgan, Iran \\ ${ }^{7}$ Department of General Medicine, Guilan University of Medical Sciences, Rasht, Guilan, Iran \\ ${ }^{8}$ Student Research Center, School of Medicine, Iran University of Medical Sciences, Tehran, Iran \\ ${ }^{9}$ Universal Scientific Education and Research Network (USERN), Tehran, Iran \\ Corresponding Author*: Ali Sadeghinia*, Assistant professor of Dermatology, Autoimmune Bullous Disease Center, Razi Hospital, Tehran University \\ of Medical Sciences, Tehran, Iran \& Department of Dermatology, Razi Hospital, Tehran University of Medical Sciences, Tehran, Iran
}

\begin{abstract}
Background: The incidence of cutaneous malignant melanoma (CMM) has increased continuously during recent decades among many populations of the world. Cutaneous Malignant Melanoma is a malignancy with a demographic and ethnic disparity located in the skin and mucous membranous. The objective of this study is to determine Cutaneous Melanoma (CM) characteristics in Iran.

Methods: This is a retrospective cross sectional study. We used data from Razi Hospital, Tehran University Melanoma Cancer Registry. The cases included in this study met the following criteria: primary CM, in situ or invasive, diagnosed in the recent past ten years. We have evaluated prognostic factors in addition to basic demographic data.

Result: A total of 193 melanomas (99 males (51.3\%) and 94 females (48.7\%)) were included. The mean age was $58.3 \pm 15.8 y e a r s$. The most frequent location for melanoma was lower extremity (75 patients, $38.9 \%)$. The most frequent subtype for melanoma based on histopathology was the Acral Lentiginous Melanoma (ALM) (74 patients, $38.3 \%)$. There was statistically significant difference between histological subtypes of melanoma and Breslow thickness $(p<0.001)$.

Conclusion: Present study showed risk factors such as age and pre-existing nevus are important factors in CMM incidence in Iran. Early detection of melanoma is a key factor in improving patients' survival.

Keywords: Cutaneous Melanoma, Malignant Melanoma, Cancer, Epidemiology, Histopathology, Immunohistochemistry, Histology, IHC, Original study, Skin cancer
\end{abstract}

\section{INTRODUCTION}

Melanoma, as a disease entity, was first described in 1812 by the Rene' Laennec [1]. The incidence of cutaneous malignant melanoma (CMM) has increased continuously during recent decades among many populations of the world [2, 3]. While a decrease of mortality rates have been observed in a number of countries due to the early detection of thinner cutaneous melanoma [4]. Cutaneous malignant melanoma is a malignancy with a demographic and ethnic disparity located in the skin and mucous membranous [5-7]. Risk factors for Malignant Melanoma(MM) include Ultraviolet Radiation (UVR), phenotype (light hair /eye /skin color with freckles), positive family history of first-degree relatives diagnosed with melanoma, DNA repair defects (e.g. Xeroderma Pigmentosum), congenital nevus, multiple acquired common or atypical melanocytic nevi, a personal history of Cutaneous Melanoma(CM)and immunosuppression $[4,6,6$, 9]. Genetic factors include: pigmentation genes named melanocortin 1 receptor (MC1R), BRAF mutations (associated particularly with excessive sunlight exposure in youths) and NRAS mutations (among older individuals particularly with continuous, intermittent sun exposure over a lifetime) [8], KIT Mutations (with cumulative sun-induced damage) and mutations in Gproteins of the Gaq family of GTPases.
Different types of primary cutaneous melanoma include Superficial Spreading Melanoma (SSM), Nodular Melanoma (NM), Lentigomaligna Melanoma (LMM) and Acral Lentiginous Melanoma (ALM). The melanomas are frequently seen on the back and legs followed by the head and neck, and anterior trunk [10].

The strongest predictors of mortality which is also included in the American Joint Committee on Cancer (AJCC) staging is Breslow depth or tumor thickness [2]. Other components necessary for histopathologic reports according to AJCC classification are ulceration, mitoses $/ \mathrm{mm}^{3}$ (for and regression $[10,11]$.

In Iran, most of cases of Nonmelanoma Skin Cancer and $\mathrm{MM}$ occur mainly because of direct exposure of patients to sun and inadequate use of sunscreens and protective hats [6]. Research studies of skin cancer in Iran have been limited. The objective of this study was to determine CM characteristics in Iran.

\section{MATERIAL AND METHODS}

This is a retrospective cross sectional study. We used data from Razi hospital, Tehran University Melanoma Cancer Registry. The cases included in this study met the following criteria: primary $\mathrm{CM}$, in situ or invasive, and diagnosed in the recent past ten years. The number of cases meeting all inclusion criteria was one hundred and ninety-three 
patients. The following clinical variables were analyzed: gender, age at the time of diagnosis, anatomic location (head and neck, trunk, upper limbs, lower limbs, acral regions and others), atypical nevi and solar lentigo, personal history of non-melanoma skin cancer and their families, family history of $\mathrm{CM}$, non-cutaneous cancer, histologic subtype, history of radiotherapy, history of tanning bed use or PUVA therapy, history of radiodermatitis and its distance from the site of the tumor, underlying diseases predisposing to melanoma. We evaluated prognostic factors in addition to basic demographic data (tumor thickness, lymphadenopathy).

The histological subtype type includes Superficial Spreading Melanoma (SSM), Nodular Melanoma (NM), Acral Lentiginous Melanoma (ALM), Lentigomaligna and Lentigomaligna Melanoma (LM/LMM) and others. We also evaluate tumor thickness and Clark level.

Data Analysis: For comparing two independent categorical variables Chi-squared test and if needed Fisher exact test was used. Kruskal Wallis nonparametric test with Dunn Post hoc comparison test was used to compare several ordinal variables and finally independent sample t test was used to compare mean of two quantitative variables. A significance level of 0.05 was considered statistically significant. All statistical analyses were performed with the Statistical Package for Social Sciences (SPSS) version 20. This study was approved by the Research Ethics Committee of Tehran University.

\section{RESULTS}

A total of 193 melanomas (99 males (51.3\%) and 94 females (48.7\%)) were included. The mean age was $58.3 \pm 15.8$ years. The minimum and maximum of age was 13 and 87, respectively. Women with melanoma outnumber men by at least 1.05 to 1 . The most frequent location for melanoma was lower extremity (75 patients, 38.9\%), followed by the head and neck (72 patients, 37.3\%), upper extremity (36 patients, 18.7\%), and trunk (10 patients, $5.2 \%)$.

The most frequent subtype for melanoma based on histopathology was the ALM (74patients, 38.3\%), followed by the LMM (55 patients, 28.5\%), NM (46 patients, 23.8\%), and SSM (18 patients, 9.3\%). (Figure 1).

There was statistically significant difference between histological subtypes of melanoma and Breslow thickness $(p<0.001)$ table 1. Such that there are significant differences between subtype of LMM with ALM $(P=0.004)$ and LMM with NM $(P<0.001)$ and SSM with NM $(P=0.014)$ Table 1. The Breslow was thicker in nodular and acral lentiginous melanoma than in the other subtypes. Personal and family history of cutaneous melanoma and other cancers were assessed. The personal history of melanoma was positive in 11 patients (5.69\%), NMSC (5patients, $2.59 \%$ ) while history of other non-skin cancers was positive in 2 patients (1.03\%). The family history of melanoma was also positive in 3 patients (1.55\%). There was no history of Epidermodysplasia verruciformis and actinic keratosis. One patient had xeroderma pigmentosum and ten patients had lentigo (10patients, 5.18\%). The minimum and maximum diameter was $5 \mathrm{~mm}$ and $6 \mathrm{~cm}$, respectively. The mean tumor diameter was $2.78 \pm 1.63 \mathrm{~cm}$.
Melanoma had arisen in a pre-existing nevus in 54 patients $(27.97 \%), 45$ patients $(23.35 \%)$ had one nevus and there were multiple or dysplastic nevus in 9 patients (4.7\%). Adenopathy was seen in 32 patients (16.6\%).

They included 58 cases of melanoma with Breslow thickness 1-2mm (30.1\%), 55patients $(2-4 \mathrm{~mm}$, $28.5 \%)$, and 43 (>4mm, 22.3\%) and $37(<1 \mathrm{~mm}, 19.2 \%)$. There was statistically significant difference between histological subtypes of melanoma and Clark's level $(p<0.001)$. To the extent that there was significant difference between LMM with ALM (P-value $=0.001)$ and LMM with NM (P-value<0.001) and SSM with NM ( $P$ value $=0.015)$ Table 1 . The most frequent pathology in head and neck melanoma was LMM followed by the ALM in upper and lower extremities and trunk in NM Figure 2. There was statistically significant difference between histological subtypes of melanoma and number of melanocytic nevus (number of mole) $(P=0.012)$, thus and so there is significant difference between ALM and LMM (P-value $=0.014)$ Table 1. The most pathologic subtype of melanoma superimposed on nevus was NM, LMM, and ALM, respectively.

We observed significant differences between mean diameter of LMM and NM histopathology $(P$-value $=0.003$, mean \pm SD: $2.11 \pm 1.32,4.3 \pm 1.5$, respectively).

There was statistically significant relationship between histopathology and adenopathy $(p=0.004)$ Figure 3 , where there is significant relationship between NM type of pathology and ADP (P-value $<0.001, \mathrm{OR}=4.4,95 \% \mathrm{Cl}$ : $1.97 \pm 9.7)$ to the extent that the odds of positive ADP in NM type of pathology is 4.4 times higher than of other type of pathologies. Furthermore, NM and ALM was the most pathology that had adenopathy, respectively.

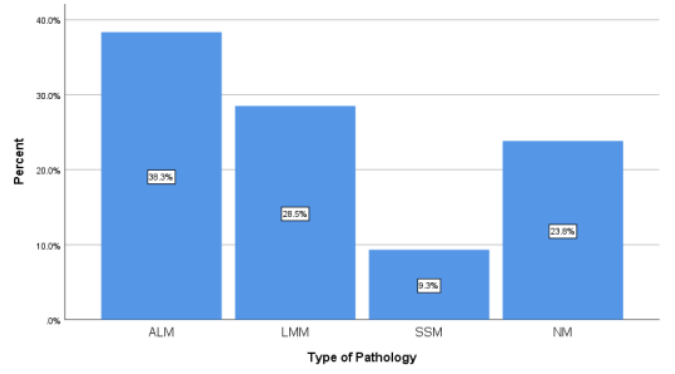

Figure 1. This Figure shows frequency of each subtype of melanoma by percentage in this study.

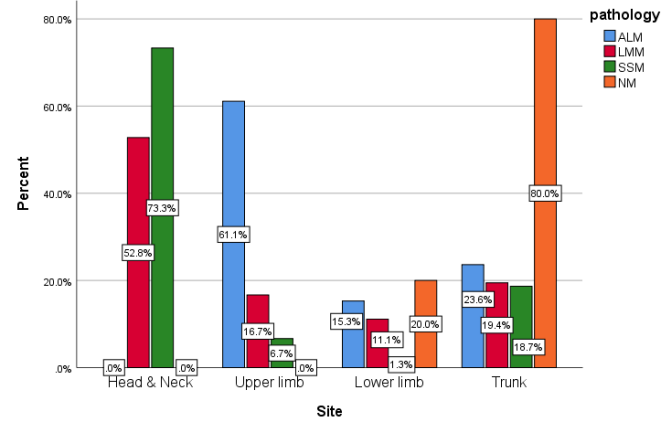

Figure 2. Site of melanoma and its subtype in study population 


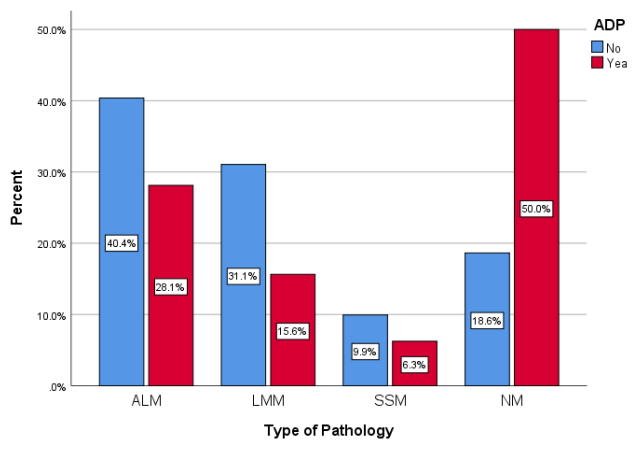

Figure 3. Cluster bar plot of types of melanoma and the ADP

Table 1. Comparison of Breslow and Clarck thickness in different histological subtypes of melanoma

\begin{tabular}{|c|c|c|c|c|}
\hline 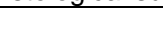 & pathology & $\mathrm{N}$ & Mean Rank & P-value* \\
\hline \multirow[t]{4}{*}{ Breslow } & ALM & 74 & 102.66 & \multirow{4}{*}{$<0.001$} \\
\hline & LMM & 55 & 70.09 & \\
\hline & SSM & 18 & 80.72 & \\
\hline & NM & 46 & 126.43 & \\
\hline \multirow{4}{*}{ Clark } & ALM & 72 & $101 / 13$ & \multirow{4}{*}{$<0 / 001$} \\
\hline & LMM & 53 & $65 / 27$ & \\
\hline & SSM & 18 & $79 / 72$ & \\
\hline & NM & 45 & $124 / 23$ & \\
\hline \multirow{4}{*}{$\begin{array}{l}\text { Number of } \\
\text { Mole (PMH) }\end{array}$} & ALM & 74 & $84 / 04$ & \multirow{4}{*}{ 0/012 } \\
\hline & LMM & 55 & $107 / 78$ & \\
\hline & SSM & 18 & $100 / 67$ & \\
\hline & NM & 46 & $103 / 52$ & \\
\hline
\end{tabular}

${ }^{*}$ Kruskal Wallis nonparametric test

\section{DISCUSSION}

In Iran, exposure to sun among people and unawareness of melanoma accounts for the increase in the incidence of melanoma. This article design to evaluate epidemiology and histology of melanoma in patients registered to Razi Hospital, Tehran, Iran in during a ten-year period.

In our study the incidence of cancer was almost similar in men and women. This was similar to the reports of Fuglede et al and Katalinic et al from Denmark and Germany [12, 13]. The incidence of melanoma is different on the basis of sex among of various countries such as countries in North America.[14]

The most common location of the tumor in our study was lower limb followed by head and neck, upper limb and trunk, respectively. In a study by anger et al in Brazil the most common location was trunk in men and lower limb in women [15]. In another study by Nagore et al in Spain the trunk was the most common site of involvement [16]. The main sites in Caucasian patients are trunk and shins based on different reports [14].

Oumeish et al from Jordan, Osterlind et al from Denmark, Nagore et al reported the most common pathology of tumor is SSM. This finding is in contrast to our study that the most common pathology of tumor was ALM followed by LMM and NM, respectively [16-18].

The Breslow thickness (the most important prognostic factor in $\mathrm{CM}$ ) was $1-2 \mathrm{~mm}$ in majority of patients. There was significant difference between Breslow thickness and pathology as thicker tumors was seen in NM and ALM. The lowest Breslow thickness was reported in SSM cases. The Breslow thickness of CM has been reported in few previous studies. In a study performed in Spain [19] the mean Breslow thickness was $2.63 \mathrm{~mm}$ which is similar to our results.

The tumor invasion was mainly at Clark level III in the time of diagnosis and the Breslow thickness was mostly 1-2 $\mathrm{mm}$. Based on the obtained data of this study, the most patients in Iran are diagnosed in higher stages. On reason for this finding may be related to the most common subtype of CM (ALM) in Iran because of difficult and delayed diagnosis and also misdiagnosis.

In the study by Marghoob et al in New York University melanoma surveillance decreased with increasing tumor thickness and Clark level so there was significant difference between Breslow thickness and Clark level increase as our study [20]. Adenopathy was only seen in $16.6 \%$ of cases almost in line with Nagore study (17.8\%) [16].

According to new studies genome pathways is effective factor in incidence of CM but in our study the family history of CM was only positive in $1.55 \%$ of the patients and incontestable in comparison to other cancers in patients and their family. Although in many studies actinic keratoses, xerotherma pigmentosum and lentigo have been mentioned important risk factors for the development of cutaneous melanoma, [21] our investigation results were not confirming that.

In a study from Switzerland, dysplastic nevus was seen in $18 \%$ of general population and $56 \%$ of cases with $\mathrm{CM}$ had dysplastic nevus that indicate, dysplastic nevus is a risk factor for CM. In our study few cases suffered from a tumor, super imposed on dysplastic nevus. In another study by Bataille et al, atypical nevus was the strongest factor in CM development and common and non dysplastic nevus were also reported to be important risk factors [22]. In our study, $27.7 \%$ of patients had preceding nevus, that may be accounted as a noteworthy factor in $\mathrm{CM}$ incidence.

A study for comparing population-based incidence data from five Iranian provinces with rates in the states of the United States(US) while standardizing ambient UVR showed remarkably lower incidence rates of melanoma in Iranian provinces with similar UVR exposures. This underscore the need for additional comparative studies to investigate the influence of other intrinsic and extrinsic factors on the risk of this malignancy [23].

A Retrospective 10-Year Study from Iran showed incidence of melanoma was higher in housewives among women and farmers among men. It was also more common in lower limbs [24].

Limitations: This study was limited by its retrospective nature and the findings require validation in a larger prospective cohort. We also lack information on genetic susceptibility.

\section{CONCLUSION}

Present study showed risk factors such as age and preexisting nevus are important factors in CM incidence in Iran. The factors such as genetic and family history of melanoma did not have a great role in tumor incidence. Most of the patients in our hospital had higher tumoral stages, more thickness and more tumoral invasion. Early detection of melanoma is a key factor in improving patients' 
survival. Therefore, this study suggests any change in a pigmented skin lesion over the course of months or years is important for melanoma diagnosis.

Acknowledgement: The authors would like to thank Dermatopathology Department of Razi Hospital for giving access to their archive. Also the authors would like to express their gratitude to the staff of the Rasool Akram Medical Complex Clinical Research Development Center (RCRDC) for their technical and editorial assistance.

Financial Support and Sponsorship: None to declare.

Conflict of Interest: There are no conflicts of interest.

\section{REFERENCES}

1. Whiteman DC, Pavan WJ, Bastian BC. The melanomas: a synthesis of epidemiological, clinical, histopathological, genetic, and biological aspects, supporting distinct subtypes, causal pathways, and cells of origin. Pigment Cell Melanoma Res. 2011;24(5):879-897.

2. Kruijff $S$, Bastiaannet $E$, Francken $A B$, Schaapveld $M$, van der $\mathrm{Aa}$ M, Hoekstra HJ. Breslow thickness in the Netherlands: a population-based study of 40880 patients comparing young and elderly patients. $\mathrm{Br} J$ Cancer. 2012;107(3):570-574.

3. Lasithiotakis $\mathrm{KG}$, Leiter $\mathrm{U}$, Gorkievicz $\mathrm{R}$, Eigentler $\mathrm{T}$, Breuninger H, Metzler G, Strobel W, Garbe C. The incidence and mortality of cutaneous melanoma in Southern Germany: trends by anatomic site and pathologic characteristics, 1976 to 2003. Cancer. 2006;107(6):1331-1339.

4. Leclerc-Mercier S, Bodemer C, Michel B, Soufir N, BourdonLanoy E, Frassatti-Biaggi A, Delanoe P, Fraitag S, HadjRabia S. Melanoma in xeroderma pigmentosum type $C$ children: Overrepresentation of desmoplastic type? J Am Acad Dermatol. 2015;72(6):e173-176.

5. Chi Z, Li S, Sheng X, Si L, Cui C, Han M, Guo J. Clinical presentation, histology, and prognoses of malignant melanoma in ethnic Chinese: a study of 522 consecutive cases. BMC Cancer. 2011;11:85.

6. Noorbala MT, Mohammadi S, Noorbala M. Cutaneous malignant melanoma in central iran: a 20-year study. Iran Red Crescent Med J. 2013;15(8):690-694.

7. Yaghoobi R, Pazyar N, Kalantar H, Nikoo A, Naraghi Z, Kamyab K, Ganadan A, Khodashenas Z, Goodarzi A Mohaghegh F. Diagnostic concordance among dermatopathologists in basal cell carcinoma subtyping: Results of a study in a skin referral hospital in Tehran, Iran. Iranian Journal of Dermatology. 2017;20(1):21-25.

8. Berwick M, Erdei E, Hay J. Melanoma epidemiology and public health. Dermatol Clin. 2009;27(2):205-214, viii.

9. Kamyab-Hesari K, Seirafi H, Jahan S, Aghazadeh N, Hejazi P, Azizpour A, Goodarzi A. Nevus sebaceus: a clinicopathological study of 168 cases and review of the literature. International journal of dermatology. 2016;55(2):193-200.

10. Societies IFoPC, Research SfM. Pigment cell \& melanoma research. Oxford : Blackwell Munksgaard. 2008.
11. Dummer R, Hauschild A, Guggenheim M, Keilholz U, Pentheroudakis G. Cutaneous melanoma: ESMO Clinical Practice Guidelines for diagnosis, treatment and follow-up. Ann Oncol. 2012;23 Suppl 7:vii86-91.

12. Fuglede NB, Brinck-Claussen U, Deltour I, Boesen EH, Dalton SO, Johansen C. Incidence of cutaneous malignant melanoma in Denmark, 1978-2007. Br J Dermatol. $2011 ; 165(2): 349-353$.

13. Katalinic A, Kunze U, Schäfer T. Epidemiology of cutaneous melanoma and non-melanoma skin cancer in SchleswigHolstein, Germany: incidence, clinical subtypes, tumour stages and localization (epidemiology of skin cancer). $\mathrm{Br} J$ Dermatol. 2003;149(6):1200-1206.

14. MacKie RM, Hauschild A, Eggermont AM. Epidemiology of invasive cutaneous melanoma. Ann Oncol. 2009;20 Suppl 6(Suppl 6):vi1-7.

15. Anger M, Friedhofer H, Fukutaki M, Ferreira M, Landman G. Primary Cutaneous Melanoma: An 18-Year Study. Clinics (São Paulo, Brazil). 2010;65:257-263.

16. Nagore E, Oliver V, Botella-Estrada R, Moreno-Picot S, Guillén C, Fortea JM. Clinicopathological analysis of 1571 cutaneous malignant melanomas in Valencia, Spain: factors related to tumour thickness. Acta Derm Venereol. 2006;86(1):50-56.

17. Osterlind A, Hou-Jensen K, Møller Jensen O. Incidence of cutaneous malignant melanoma in Denmark 1978-1982. Anatomic site distribution, histologic types, and comparison with non-melanoma skin cancer. $\mathrm{Br} J$ Cancer. 1988;58(3):385-391.

18. Oumeish OY. Epidemiology of primary cutaneous malignant melanoma in Jordan. Int J Dermatol. 1997;36(2):113-115.

19. Arranz-Sánchez DM, Pizarro Á, Valencia-Delfa JL, VilletaLópez M, González-Beato MJ, Mayor-Arenal M, CasadoJiménez M. Clinical-Pathological Features of Cutaneous Melanomas Diagnosed in a Mediterranean Tertiary Hospital Between 1990 and 2004: A Comparison Between Sexes and Age Groups and Analysis of Long-Term Outcomes. Actas Dermo-Sifiliográficas (English Edition). 2009;100(6):476-485.

20. Marghoob AA, Koenig K, Bittencourt FV, Kopf AW, Bart RS. Breslow thickness and clark level in melanoma: support for including level in pathology reports and in American Joint Committee on Cancer Staging. Cancer. 2000;88(3):589-595.

21. Meyle KD, Guldberg P. Genetic risk factors for melanoma Hum Genet. 2009;126(4):499-510.

22. Bataille V, Grulich A, Sasieni P, Swerdlow A, Newton Bishop $J$, McCarthy W, Hersey P, Cuzick J. The association between naevi and melanoma in populations with different levels of sun exposure: a joint case-control study of melanoma in the UK and Australia. $\mathrm{Br} J$ Cancer. 1998;77(3):505-510.

23. Moslehi R, Zeinomar N, Boscoe FP. Incidence of cutaneous malignant melanoma in Iranian provinces and American states matched on ultraviolet radiation exposure: an ecologic study. Environ Pollut. 2018;234:699-706.

24. Ferdosi S, Saffari M, Eskandarieh S, Raziyeh $R$, Moghaddam MG, Ghanadan A, Shirkoohi R. Melanoma in Iran: a Retrospective 10-Year Study. Asian Pac J Cancer Prev. 2016;17(6):2751-2755. 